\title{
GENERAIJZED IIPPMANN-SCHWINGER SCATTERING EQUATIONS
}

FOR SINGULAR INTERACTIONS

\author{
W.Güttinger * \\ CERN - Geneva \\ E. Pfaffelhuber $\left.{ }^{* *}\right)$ \\ Institute of Theoretical Physics, \\ University of Munich
}

$A B S T R A C T$

The Iippmann-Schwinger equation is generalized to singular scattering amplitudes coming from unrenormalizable field theories, marginally singular interactions and from singular potentials. The generalization is based on an extension of the Feynman prescription to singular input functions and on a corresponding extension of the potential concept. The connection of the generalized Lippmann-Schwinger equation with partial wave equations analytically continued in the angular momentum is established. The equation makes sense for repulsive and attractive interactions and also in those cases where no analytic continuation in the angular momentum is possible. Some properties of the generalized Iippmann-Schwinger equation are discussed and a solution method is outlined. A contour integral representation for singular input functions is deduced.

\footnotetext{
*) On leave from the University of Munich.

**) Fellow of the Deutsche Forschungsgemeinschaft.
} 


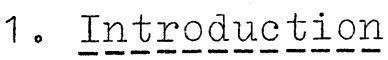

In this paper we discuss a generalized IippmanSchwinger equation for singular scattering amplitudes coming from unrenormalizable field theories, marginally singular interactions typical of vector bosons and $\phi^{4}$ theory and from singular potential scattering. The conventional IippmannSchwinger equation 1 does not exist in these cases because the Fourier transform of the interaction kernel diverges (there does in general not even exist a Born term) 2, 3. Nevertheless, scattering amplitudes singular at zero coupling and possessing an essential singularity at infinite momentum have been obtained for various strongly repulsive interactions by introducing auxiliary cutoffs 2,3,4, by a cutoff-free peratization 5 or by passing to differential equations in momentum space ${ }^{6}$. We shall show that the question for the scattering equations satisfied by singular amplitudes leads to a generalization of the Feynman prescription to singular input functions and, as a special case, to an extension of the potential concept. Being deducible from first principles in case of repulsive interactions, these generalizations also make sense in the attractive case.

In section 2 we introduce the concept of "regular" potentials $V_{R}$ equivalent to singular ones, $V, V_{R}$ differing from $\mathrm{V}$ by a multipole core localized at the origin which kills the singularity of $V$ at $r=0$. At no stage of our considerations will a cutoff be involved. In Sections 3 and 4 we deduce a generalized Iippmann-Schwinger (IS) equation for singular repulsive potentials and extend it to the attractive case: We show, in 
particular, how this equation is related to partial wave equations analytically continued in the angular momentum variable. Our equations also make sense in those cases where no such continuation is possible. Finally, we discuss in section 5, a generalized relativistic Iippmann-Schwinger equation for the $T$ matrix of unrenormalizable and $\phi^{4}$ theories. This equation operates directly in Minkowski space and reduces to equations analogous to those of singular potertial scattering if a rotation to Euclidean metric can be done. Some properties of the equations are discussed, in particular, the appearance of a scaling parameter and of a characteristic length. A method for solving the IS equations is outlined in Sec.6. From the technical point of view our considerations are based upon a contour integral representation for singular interaction kernels. 


\section{Equivalent Potentials}

Let us consider a potential $V(r)$ singular at the origin $r=0$. We shall call a function $V_{R}(r)$ a "regular potential equivalent to $V(r)$ " if the following two conditions are satisfied:

(i) $V_{R}(r)$ coincides with $V(r)$ everywhere except at $r=0$ 。 That is to say, the relation $[\hat{1}]$

$$
\int_{-\infty}^{\infty} d \vec{r} V_{R}(r) \varphi(\vec{r})=\int_{-\infty}^{\infty} d \vec{r} V(r) \varphi(\vec{r})
$$

holds for all those test functions $\varphi(\vec{r})$ which vanish at $r=0$ together with a sufficiently large number of derivatives so that $\int_{-\infty}^{\infty} \overrightarrow{\mathrm{r}} \mathrm{V}(r) \varphi(\vec{r})$ exists. In particular, if $\mathbb{M}$ is the smallest non-negative integer such that $V(r) r^{2 M+2}$ is integrable at $r=0$, the relation $(2.1)$ holas for all $\varphi(\vec{r})$ with $\partial^{n} \varphi(0) / \partial x_{i}{ }^{n}=0$, $\mathrm{n}=0,1,2, \ldots, 2 \mathrm{M}, \quad\left(\vec{r}=\left(x_{i}\right), i=1,2,3\right)$.

(ii) $V_{R}(r)$ has a fini'e Fourier tran form $V_{R}(k)$.

By these conditions $V_{R}(r)$ is determined up to a linear combination of derivatives of $\delta(\vec{r})$,

$$
F(\vec{r})=\sum_{0}^{\mathbb{N}} c_{n} \Delta^{n} \delta(\vec{r})
$$

with arbitrary finite coefficients $c_{n}, \Delta$ being the Laplacian. Hence, if $V_{R}$ is a special potential satisfying (i) and (ij), the most general regular potential equivalent to $V$ is given by $V_{R}+F$.

The physical meaning of $V_{R}$ will become obvious from the following explicit representation. Let $p(\vec{r})$ be any test function, not necessarily vanishing at $r=0$, and let us assume that $V(r)$ decreases at infinity faster than some fixed power of $1 / r$. Then 
we can construct $V_{R}$ in accordance with the formula

$$
\int_{-\infty}^{\infty} d \vec{r} v_{R}(r) \varphi(\vec{r})
$$

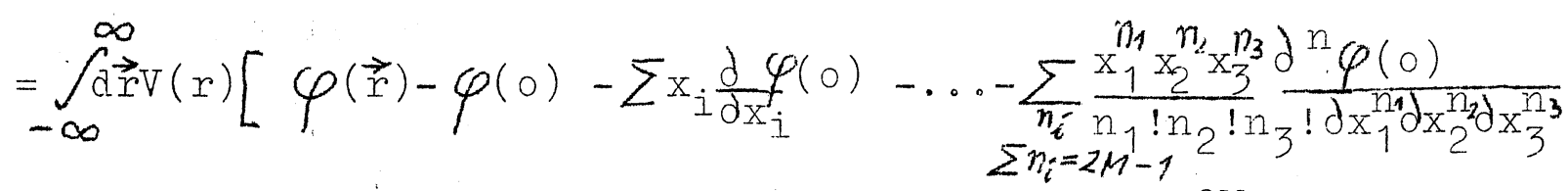

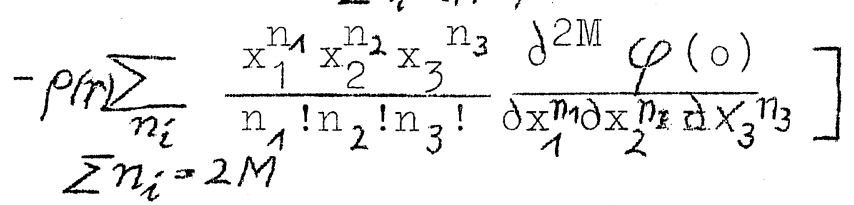

where we just subtracted enough terms of the raylor series of $\varphi$ to render the integral on the r.h.s. convergent at $r=0$. We adopt the convention that the subtraction terms are to be dropped if $V$ is regular at $r=0, i \circ e$, if $V$ is integrable at the origin and consequently $\mathrm{M}$ can formally take values less than zero. The last subtracted Taylor term (with $\sum n_{i}=2 M$ ) has been mitiplied with a factor

$$
\rho(r)= \begin{cases}\theta(\underset{m}{a-r)} & \text { if } V \sim r^{-3-2 m} \log \mu_{r}(m=0,1,2, \ldots) \text { as } r \rightarrow \infty \\ 1 & \text { in all other cases }\end{cases}
$$

where $\theta$ is the step function and $\underset{m}{a}$ is an arbiurary positive constant of dimension of length. This factor $p$ must be inserted if and only if $V$ behaves at infinity as $r^{-3-2 m} 10 g^{\mu} r$, for otherwise the integral over the last Taylor term would diverge at infinity (the odd power being decisive, not the logarithm!). If $V$ decreases at infinity in any other way we put $p=1$, i.e., subtract an ordinary finite Taylor series.

To put eq.(2.3) into compact form let us denote by

$$
\phi(r)=\frac{1}{4 \pi} \int a \omega \varphi(\vec{r})=\int d \omega \varphi(r \omega)
$$

the mean value of $\varphi(\vec{r})$ on the sphere of radius $r$ where $\overrightarrow{a r}=r^{2}$ draw. Since

$$
\phi(r)=\frac{\sqrt{\pi}}{2} \sum_{n=0}^{M} \frac{r^{2 n} \Delta^{n} \varphi(0)}{2 n n ! \Gamma(n+3 / 2)} \quad+o\left(r^{2 M}\right)
$$


we obtain from eq.(2.3) the following representation for $\mathrm{V}_{\mathrm{R}}[2]$ :

$$
\begin{aligned}
& \int_{-\infty}^{\infty} \overrightarrow{\mathrm{d}} V_{R}(r) \varphi(\vec{r}) \\
= & 4 \pi \int_{0}^{\infty} \mathrm{arr}^{2} V(r)\left[\phi(r)-\frac{\sqrt{\pi}}{2} \sum_{n=0}^{M-1} \frac{r^{2 n} \Delta^{n} \varphi(0)}{2^{2 n} n ! \Gamma(n+3 / 2)}-\frac{\sqrt{\pi}}{2} \rho(r) \frac{r^{2 M} \Delta^{M} \varphi(0)}{2^{2 M} \mathbb{M} !(M+3 / 2)^{-1}} .\right.
\end{aligned}
$$

The potential $V_{R}$ defined in this way obviously satisfies condition ( $i$ ) and $V_{R}=V$ if $V$ is regular at $r=0$. $V_{R}$ also satisfies condition ( $i i)$, for the finite Fourier transform $V_{R}(k)$ follows from eq. $(2.7)$ by substituting $(2 \pi)^{-3} \exp (i \vec{k} \vec{r})$ for $\varphi(\vec{r})$ on both sides of that equation:

$$
V_{R}(k)=\frac{1}{(2 \pi)^{3}} \int_{-\infty}^{\infty} \vec{r} V_{R}(r) \exp (i \vec{k} \vec{r})
$$

where $k=|\vec{k}|$.

Let us consider the particularly important singular potential

$$
\mathrm{V}=\mathrm{V}^{\lambda, \mu}(r)=g r^{\lambda} \log { }^{\mu}(\alpha r)
$$

where $\lambda$ is any complex number, $\mu=0,1,2, \ldots, \log \mu_{z}=(\log z){ }^{\mu}$, $g$ is a coupling constant and $\alpha$ is a given constant with dimension of a reciprocal length. $V \lambda \mu$ is singular if $\operatorname{Re} \lambda \leqslant-3 .[3]$ One easily sees that now

$$
\mathbb{N}=[-(\operatorname{Re} \lambda+3) / 2]
$$

is the largest non-negative integer less than or equal to $-(\operatorname{Re} \lambda+3) / 2, p(r)=e(\underset{m}{a}-r)$ if $\lambda=-3-2 m(m=0,1,2, \ldots)$ and $\rho(r)=1$ in all other cases. Writing the integral far in eq.(2.7) as $\lim _{\varepsilon \rightarrow 0} \int_{\varepsilon}^{\infty} d r$ we obtain for the regular potential $\mathrm{V}_{\mathrm{R}}=\mathrm{V}_{\mathrm{R}}^{\lambda \mu} \begin{gathered}\varepsilon \rightarrow 0 \\ \text { equivalent }\end{gathered}$ to $\mathrm{V}^{\lambda \mu}$ the formula 


$$
\int_{-\infty}^{\infty} \vec{d} v_{R}^{\lambda \mu}(r) \varphi(\vec{r})=\lim _{\varepsilon \rightarrow 0}\left[\int_{D}^{\infty} \vec{r} \varphi(\vec{r}) V^{\lambda \mu}(r)+\sum_{n=0}^{M} a_{n}(\varepsilon) \Delta^{n} \varphi(0)\right]
$$

or

$$
V_{R}^{\lambda \mu}(r)=\lim _{\varepsilon \rightarrow 0}\left[V^{\lambda \mu}(r) \theta(r-\varepsilon)+\sum_{n=0}^{M} a_{n}(\varepsilon) \Delta^{n} \delta(\vec{r})\right]
$$

where

$$
a_{n}(\varepsilon)=g \frac{2 \pi^{3 / 2}}{\alpha^{\lambda} 2^{2 n} n ! \Gamma(n+3 / 2)}\left[\frac{a^{\mu}}{d \sigma \mu} \frac{\alpha^{\sigma} \varepsilon^{2 n+\sigma+3}}{2 n+\sigma+3}\right]_{\sigma=\lambda}
$$

with $n=0,1,2, \ldots, \mathbb{M}$ if $\lambda \neq-3-2 m, n=0,1,2, \ldots, M-1$ and

$$
a_{M}(\varepsilon)=g \frac{2 \pi^{3 / 2}\left[\log \mu^{\mu+1}\left(\alpha_{m}\right)-\log ^{\mu+1}(\alpha \varepsilon)\right]}{2^{2 M_{M} ! \Gamma(N+3 / 2)(\mu+1)}}
$$

if $\lambda=-3-2 \mathrm{~m}$. We have that $v_{\mathrm{R}}^{\lambda \mu}=\mathrm{V}^{\lambda \mu}$ if $\operatorname{Re} \lambda>-3$.

Eqs.(2.11) and (2.12) exhibit clearly the physical meaning of $V_{R}$ : The subtracted multipole core $\sum_{n=0}^{M} a_{n}(\varepsilon) \Delta^{n} \delta(\vec{r})$ just compensates the singularity of $V(r)$ at $r=0$ thus rendering $V_{R}$ integrable at the origin. It should be emphasized that the limit $\varepsilon \rightarrow 0$ has absolutely nothirg to do with a cutoff procedure. Instead, the subtracted multipole core resembles a renormalization term. According to (2.8) we obtain the Fourier transform of $V_{R}^{\lambda \mu}(r)$ by substituting $(2 \pi)^{3 / 2} \exp (i \vec{k} \vec{r})$ for $\varphi(\vec{r})$ into eq. $(2 \cdot 11)$ :

$$
V_{R}^{\lambda \mu}(k)=\frac{g}{\pi^{3 / 2} \alpha^{\lambda}}\left[\frac{a^{\mu}}{d \sigma \mu}(2 \alpha)^{\sigma} \Gamma\left(\frac{\sigma+3}{2}\right) k^{-\sigma-3} / \Gamma(-\sigma / 2)\right]_{\sigma=\lambda}
$$

if $\lambda \neq-3-2 m$ and 
$V_{R}^{\lambda \mu}(k)=\frac{g \alpha^{2 m}}{23 \pi^{3 / 2}}\left(-\frac{1}{2}\right)^{\mu}\left[\frac{a^{\mu}}{\partial \sigma \mu} \frac{(-1)^{\sigma+1}(k / 2 \alpha)^{2 \sigma}}{\sigma ! T(\sigma+3 / 2)}\left\{\operatorname{Iog}\left(\frac{a^{2} k^{2}}{4}\right)-\psi(\sigma+1)\right.\right.$ $-\psi(\sigma+3 / 2)\}] \sigma=m$

if $\lambda=-3-2 \mathrm{~m}$, where $\psi(z)=\Gamma^{\prime}(z) / \Gamma(z)$.

To establish a connection with analytic continuation in $\lambda$ and in the angular momentum variable (cf., Secs.3 and 4) and to prepare the extension to the relativistic case (Sec.5) we prove the following theorem.

Theorem: The potential $\mathrm{V}_{R}^{\lambda \mu}(r)$ and its Fourier transform $\mathrm{V}_{\mathrm{R}}^{\lambda \mu}(\mathrm{k})$ coincide with the analytic continuation in $\lambda$ of $v^{\lambda \mu}(r)$ and $V^{\lambda \mu}(k)$, respectively, from $\operatorname{Re} \lambda>-3$ to the entire complex $\lambda$ plane except $\lambda=-3-2 \mathrm{~m}(\mathrm{~m}=0,1,2, \ldots)$. If $\lambda=-3-2 \mathrm{~m}$, then $\mathrm{V}_{\mathrm{R}}^{\lambda \mu}(\mathrm{r})$ and $\mathrm{V}_{\mathrm{R}} \lambda \mu(\mathrm{k})$ coincide with the analytic continuation in $\lambda$ of the regular parts of the Iaurent expansions of $v^{\lambda \mu}(r)$ and $v^{\lambda \mu}(k)$, respectively, around the poles $\lambda=-3-2 \mathrm{~m}$, i.e with the analytic continuation of

$$
v^{\lambda \mu}-\left(\operatorname{Res}_{\lambda=-3-2 \mathrm{~m}} \mathrm{~V}^{\lambda}\right)\left[\alpha^{\mu}\left(\alpha^{\lambda} /(\lambda+3+2 \mathrm{~m})\right) / \mathrm{d} \lambda^{\mu}\right] \alpha^{-\lambda}
$$

where $v^{\lambda} \circ=\left(v^{\lambda} \mu\right)_{\mu=0}$. This implies that

$$
\int_{-\infty}^{\infty} \overrightarrow{d r V} V_{R}(r) \varphi(\vec{r})=\frac{1}{2 \pi i} \int_{C} \frac{d z}{z}\left[\int_{-\infty}^{\infty} \vec{r} V(r)\left(\frac{a}{r}\right)^{z} \varphi(\vec{r})\right]
$$

and

$$
V_{R}(k)=\frac{1}{2 \pi i} \int \frac{d z}{z}\left[\frac{1}{(2 \pi)^{3}} \int_{-\infty}^{\infty} \operatorname{drV}(r)\left(\frac{a}{\bar{r}}\right)^{z} \cdot e^{i \vec{k} r}\right]
$$


where $\mathrm{C}$ is a small circle around $\mathrm{z}=0$ 。 Eqs.(2.16) and $(2.17)$ hold for all singular potentials $V(r)$, in particular for $V=V^{\lambda \mu}$ where $V_{R}^{\lambda \mu}$ is given by eqs. $(2.11)-(2.15)$ 。

In eqs. $(2.16)$ and $(2.17)$ we first have to calculate the integral over $r$ on the $r \circ h \circ s$. for Re $z$ sufficiently large negative (where the integral converges), then continue the resulting function of $z$ analytically to a region of the complex $z$ plane containing the origin $z=0$ and finally perform the $z$ integration. The $r$ and $z$ integrals do not commute. The resulting expressions for $V_{R}^{\lambda \mu}$ are independent of the arbitrary length $\underset{m}{a}$ if $\lambda \neq-3-2 m$.

To prove the theorem we consider first the case $\mu=0$, $\mathrm{V}=\mathrm{V}^{\lambda 0}=\mathrm{gr} \lambda$, and start from the identity

$$
\begin{aligned}
g \int_{-\infty}^{\infty} d \vec{r} r^{\lambda} \varphi(\vec{r})= & 4 \pi g \int_{0}^{a} d r r^{\lambda+2}\left[\phi(r)-\frac{\sqrt{\pi}}{2} \sum_{0}^{M} \frac{r^{2 n} \Delta^{n} \varphi(0)}{2^{2 n n !} \Gamma(n+3 / 2)}\right] \\
& +4 \pi g \int_{a}^{\infty} d r r^{\lambda+2} \phi(r) \\
& +2 \pi^{3 / 2} g \sum_{0}^{M} \frac{a^{2 n+3+\lambda} \Delta^{n} \varphi(0)}{(2 n+3+\lambda) 2^{2 n} n ! \Gamma(n+3 / 2)}
\end{aligned}
$$

valid for $\operatorname{Re} \lambda>-3$, M being given by eq.(2.10). The l.h.s. of eq.(2.18) is analytic in $\lambda$ for $\operatorname{Re} \lambda>-3$. The r.h.s. exists for all $\lambda \neq-3-2 m$ and thus defines the analytic continuation of the 1.h.s. to the entire $\lambda$ plane except $\lambda=-3-2 \mathrm{~m}$ where it has simple poles. Since for $\lambda \neq-3-2 \mathrm{~m}$

$$
\left.2 \pi^{3 / 2} \sum_{0}^{M} \frac{a^{2 n+3+\lambda} \Delta^{n} \varphi(0)}{(2 n+3+\lambda) 2^{2 n} n ! \Gamma(n+3 / 2)}=4 \pi \int_{a}^{\infty} d r \sum_{0}^{M} \frac{r^{2 n+2+\lambda} \Delta^{n} \varphi(0)}{2^{2 n} n ! \Gamma(n+3 / 2)}\right)
$$

the r.h.s. of eq. (2.18) is identical with that of eq. (2.7) for $V=V^{\lambda o}$. If $\lambda=-3-2 m$, the theorem follows by aropping the term with $n=\mathbb{M}$ in the last series of eq.(2.18) what just $65 / 1211 / 5$ 
produces the factor $p$ in eq.(2.7) . Hence, eq.(2.11) follows for $\mu=0$. Differentiating $\mu$ times with respect to $\lambda$ gives the result for $\mu \neq 0$. The statements about the Fourier transforms are then obvious. Or else, by airect calculation one sees that eq.(2.14) is true for $\operatorname{Re} \lambda>-3$ and the r.h.s. can be continued analytically to all $\lambda \neq-3-2 \mathrm{~m}$ whereas we can continue the regular part of eq.(2.14) if $\lambda=-3-2 \mathrm{~m}$. To prove eqs.(2.16) and $(2.17)$ we start from the identity $a$
$g \int_{-\infty}^{\infty} d \vec{r} r^{\lambda}\left(\frac{a}{r}\right)^{z} \varphi(\vec{r})=4 \pi g \int_{0}^{m} d r r^{\lambda+2}\left(\frac{a}{r}\right)^{z}\left[\phi(r)-\frac{\sqrt{\pi}}{2} \sum_{0}^{M} \frac{r^{2 n} \Delta^{n} \varphi(0)}{2^{2 n} n ! \Gamma(n+3 / 2)}\right]$ $+4 \pi g \int_{a}^{\infty} d r r^{\lambda+2}\left(\frac{a}{r}\right)^{z} \phi(r)+2 \pi^{3 / 2} g \sum_{0}^{M} \frac{a^{2 n+3+\lambda} \Delta^{n} \varphi(0)}{(2 n+3+\lambda-z) 2^{2 n} n ! T(n+3 / 2)}$

valid for $\operatorname{Re}(\lambda-z)>-3$ and continue the r.h.s. into a region of the $z$ plane containing the origin $z=0$. Operating on both sides of eq. (2.19) with $\frac{1}{2 \pi i} \int_{C} z / z$ leaves the two integrals on the r.h.s. unaltered since they are regular at $z=0$ while the same operation on the last series just yields the value of that series at. $z=0$ if $\lambda \neq-3-2 m$ and eliminates the pole term if $\lambda=-3-2 \mathrm{~m}$. Differentiating $\mu$ times with respect to $\lambda$ gives eqs. $(2.16)$ and $(2.17)$ for $V=V^{\lambda \mu}$. It is obvious that these equations hold for all singular potentials $V$ whatsoever. In particular, we have that

$$
\left.V_{R}^{-n, \mu}(k)=\frac{1}{2 \pi i} \frac{\hat{a}_{z}}{z} a^{z_{V}} V_{R}^{-n-z}, \mu_{k}\right)
$$

where for $V_{R}^{-n-z, \mu}(k)$ on the r.h.s. we have to irsert the expression (2.14) with $\lambda=-n-z$ (which follows directly by analytic continuation).

We wish to point out that the expression (2.11) for 
$\lambda=-3-2 \mathrm{~m}$ is not the value of the r.h.s. of eq.(2.18) at $\boldsymbol{\lambda}=-3-2 \mathrm{~m}$, for the r.h.s. of eq.(2.18) has a pole at this point. However, eq.(2.11) holds for all $\lambda$ : The subtraction term $\sum_{i}^{M} a_{n} \Delta^{n} \varphi(0)$ just compensates the value of the integral $\underset{r \geqslant \varepsilon}{\int} \vec{r} V(r) \varphi(\vec{r})$ at the lower boundary $r=\varepsilon$. The regular potential $V_{R}$ equivalent to $V$ also is known as a pseudofunction, finite part or regularization of $\mathrm{V} .5,7$

It should be emphasized that in a theory with a potential $V=g r^{-3-2 m} \log \mu^{h}(\alpha r)$ there appears an additional arbitrary length $\underset{m}{a}$ besides the given constants $g$ and $\alpha$. This length $\mathrm{m}$ also furnishes the mathematical means for rendering the r.h.s. of eq. (2.15) dimensionally correct $\left(\log \left(\mathrm{a}_{\mathrm{m}}^{2} \mathrm{k}^{2} / 4\right)\right)$. Since in a theory leading to $V=g r^{-3-2 m}$ the coupling $g$ is the only quantity with dimension of some power of length, $g=I^{\eta}$, we have to express $\underset{m}{a}$ in terms of $g: \underset{m}{a}=\Lambda g^{1 / \eta}$, $\Lambda$ being an arbitrary scaling parameter. This reflects the fact that $\mathrm{V}_{\mathrm{R}}^{\lambda}{ }^{\circ}(r)$ is not scale invariant if the length $\mathrm{m}$ is kept fixed. From $\in q .(2.12)$ it follows that

$$
V_{R}^{\lambda 0}(\Lambda r)=\Lambda^{\lambda} V_{R}^{\lambda 0}(r)+A 2 \pi^{3 / 2} g(\log \Lambda) \Delta^{m} \delta(\vec{r}) / 2^{2 m} m ! \Gamma(m+3 / 2)
$$

where $A=1$ if $\lambda=-3-2 \mathrm{~m}$ and $\mathrm{A}=0$ in all other cases. However, if $\mathrm{m}$ is kept arbitrary (what is permitted) then scaling invariance is reestablished: $V_{R}{ }^{\lambda 0}(\Lambda r)=\Lambda^{\lambda} V_{R}^{\lambda 0}(r)$. The point is simply that the integral (2.8) is not invariant under a dilatation transformation if $V_{R}=V_{R}{ }^{-3}-2 \mathrm{~m}, 0$. The arbitrariness connected with the length $\mathrm{m}^{\mathrm{a}}$ and the scaling factor $\Lambda$ remains entirely within the non-uniqueness of $V_{R}$ 
due to the possible addition of the term F, eq.(2.2), however. Substituting in eq. $(2.15) \underset{m}{a}$ by $\Lambda_{m}$ we obtain $\mathrm{V}_{\mathrm{R}}^{\lambda_{0}}(\mathrm{k}) \rightarrow \mathrm{V}_{\mathrm{R}}^{\lambda 0}(\mathrm{k})+$ const. $\mathrm{k}^{2 m} \log \Lambda$. The term const. $K^{2 m} \log \Lambda$ clearly can be amalgamated into $F(k)$. We note that there will arise no ambiguity in observable quantities due to this arbitrariness in $V_{R}-3-2 m, 0$ if the potential is repulsive whereas an arbitrary phase will enter the wave function if the potential is attractive (cf., sec.3). The above scaling property has been discovered by one of us several years ago in the study of unrenormalizable field theories. The physical meaning of the length ${ }_{m}$ remains unclear since it appears only in connection with very particular long range forces $(\lambda=-3-2 \mathrm{~m})$ of potential scattering and with logarithmic divergences. In the relativistic case, however, the scaling difficulty seems to be a rather central. point。

Finally we observe that $\mathrm{V}_{\mathrm{R}}(r)$ is not positive if $\mathrm{V}(r)$ is positive and singular. $V_{R}$ is said to be positive if $\int_{-\infty}^{\infty} \vec{d} V_{R}(r) \varphi(\vec{r}) \geqslant 0$ for $\varphi \geqslant 0$. Thus we see from eq. (2.7) that for $g>0, \mathrm{~V}_{\mathrm{R}}^{\lambda} \circ$ is not positive if $\operatorname{Re} \lambda \leqslant-3$ (in virtue of the subtracted multipole term). It should be stressed, however, that this non-positivity has nothing to do with an indefinite metric: The positive-definiteness axiom of the field theories from which such potentials originate is not violated.

We note in conclusion that $\mathrm{V}_{\mathrm{R}}$ is conditionally positive: $v_{R}(\varphi) \geqslant 0$ for all $\varphi \geqslant 0$ with $\varphi(0)=\ldots=\Delta \varphi(0)=0$ 。 
3. Generalized Lippmann-Schwinger Equation for Singular Potentials

We start from the Schrödinger equation for potential.

scattering

$$
\psi(\vec{r}, \vec{k})=e^{i \vec{k} \vec{r}}+\int_{-\infty}^{\infty} d \vec{r}^{\prime \prime} V\left(r^{\prime \prime}\right) G\left(\vec{r}^{\prime \prime}, \vec{r}, \vec{k}\right) \psi(\vec{r} ", \vec{k})
$$

with the Green's function

$$
G\left(\vec{r}^{\prime \prime}, \vec{r}, \vec{k}\right)=\frac{1}{(2 \pi)^{3}} \int_{-\infty}^{\infty} d \vec{q} \frac{e^{i \vec{q}\left(\vec{r}-\vec{r}^{\prime \prime}\right)}}{k^{2}-q^{2}-i \varepsilon}
$$

and define the (off shell) scattering amplitude $\mathrm{T}$ by

$$
T(\vec{p}, \vec{k})=\frac{1}{(2 \pi)^{3}} \int_{-\infty}^{\infty} d \vec{r} e^{-i \vec{p} \vec{r}} V(r) \psi(\vec{r}, \vec{k})
$$

There holds the following theorem:

Theorem: If $V(r)$ is singular at $r=0$ and repulsive $(g>0$, $\mathrm{V} \sim \operatorname{gr} \lambda(\log (\alpha r))^{\mu}$ as $\left.r \rightarrow 0, \operatorname{Re} \lambda<-2\right)$ then $\psi(\vec{r}, \vec{k})$ vanishes at $r=0$ together with all its derivatives,

$$
\psi(0, \vec{k})=0, d^{n} \psi(0, \vec{k}) / d r^{n}=0, n=1,2, \ldots .(3.4)
$$

To prove this, let us assume that $\psi \sim r \xi a s r \rightarrow 0$. Then eq. (3.1) shows that $\xi= \pm \infty$. But $\xi=-\infty$ has to be excluded because of the assumed existence of the r.h.s. integral in eq.(3.1).

The result (3.4) permits us to replace the repulsive singular potential $V$ in eqs.(3.1) and (3.3) by the equivalent regular potential $V_{R}$. In fact, replacing $\vec{\gamma}$ by $\vec{\gamma} /$ in eq.(2.1) and inserting $\varphi\left(\vec{r}^{\prime \prime}\right)=G\left(\vec{r}^{\prime \prime}, \vec{r}, \vec{k}\right) \psi\left(\vec{r}^{\prime \prime}, \vec{k}^{\prime}\right)$ into the equation so obtained, it follows in virtue of eq.(3.4) that 
$\int_{-\infty}^{\infty} d \vec{r}^{\prime \prime} V\left(r^{\prime \prime}\right) G(\vec{r}, \prime \vec{r}, \vec{k}) \psi(\vec{r}, \vec{k})=\int_{-\infty}^{\infty} d \vec{r}^{\prime \prime} V_{R}\left(r^{\prime \prime} G(\vec{r}, \vec{r}, \vec{k}) \psi(\vec{r}, \vec{p}, 0)\right.$

Similarly, inserting $\varphi(\vec{r})=\exp (-i \overrightarrow{p r} \vec{r}) \psi(\vec{r}, \vec{k})$ into eq. $(2.1)$ we obtain

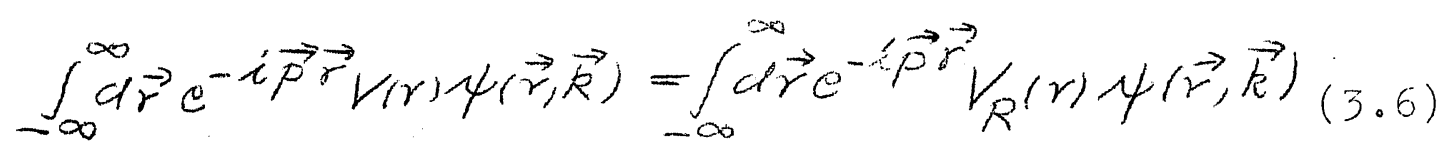

and thus

$$
T(\vec{p}, \vec{k})=\mathbb{T}_{R}(\vec{p}, \vec{k})=\frac{1}{(2 \pi)^{3}} \int_{-\infty}^{\infty} d \vec{r} e^{-i \vec{p} \vec{r}_{V_{R}}}(r) \psi(\vec{r}, \vec{k})(3.7)
$$

Inserting the expression (3.5) into equ.(3.1) and operating on the resulting Schrödinger equation with $\exp (-i \vec{p} \vec{r}) V_{R}(r)$ we obtain the generalized Iippmann-Schwinger equation for singular potentials

$$
\mathrm{T}_{R}(\vec{p}, \vec{k})=V_{R}(|\vec{p}-\vec{k}|)+\int_{-\infty}^{\infty} \vec{d} \frac{V_{R}(|\vec{p}-\vec{q}|) T_{B}(\vec{q}, \vec{k})}{k^{2}-q^{2}-i \varepsilon}
$$

where

$$
V_{R}(|\vec{p}|)=\frac{1}{(2 \pi)^{3}} \int_{-\infty}^{\infty} d \vec{r} V_{R}(r) e^{i \vec{p} \vec{r}}
$$

exist according to Sec.2. $\mathrm{Eq}$. (3.8) has been deduced for repulsive singular potentials but we shall show below and in Sec.4 that this equation also makes sense ( and indeed can be derived) in the attractive case. If $\mathrm{V}$ is regular, eq. (3.8) obviously reduces to the ordinary LS equation. If we would have proceeded formally by starting from eq.(3.1) and eq.(3.3) we would have arrived at equ. (3.8) with $V_{R}$ replaced by $V$ -- i.e. at the usual IS equation -- but this equation would have ceased to exist since $V$ does not possess a Fourier transform if it is singular.

$$
\text { If } \mathrm{V}=\mathrm{V}^{\lambda \mu} \text {, then, according to the theorem cf sec.2 }
$$
the generalized LS equation (3.8) obviously coincides with 
the analytic continuation in $\lambda$ of the conventional IS equation, valid for $\operatorname{Re} \lambda>-3$, except for $\lambda=-3-2 \mathrm{~m}$ in which case $V_{R}$ is given by eqs. $(2.12),(2.13)$ 。

The generalized IS equation has been deduced under the assumption that $V$ is repulsive. In that case we can invert the arguments: Given the Schrödinger equation (3.1) and equ.(3.8) with some function $V^{\prime}$ instead of $V_{R}(e q .(3.9))$, then it follows in virtue of eq. $(3.4),(3.5)$ and $(3.6)$ that $V^{\prime}$ must be identical with $\mathrm{V}_{\mathrm{R}}$. Therefore, the concept and the defining representation of the potential $V_{R}$ "equivalent" to $V$ follows from first principles in case of repulsive interactions.

At first sight the solution of eq.(3.8) would seem to be not unique because an arbitrary polynomial $F(p)=\sum_{0}^{M} c_{n}(-p$, $n$ (the Fourier transform of $F, e q \cdot(2.2)$ ) may be added to $V_{R}(p)$ in eq.(3.8). Actually this is not the case: The solution of eq.(3.8) is unique whatever potential $V_{R}$ equivalent to $V$ is used provided that $V$ is repulsive. Indeed, if $V_{1 R}$ and $V_{2 R}$ are two regular potentials equivalent to $\mathrm{V}$ their aifference is $V_{1 R}-V_{2 R}=F(r)=\sum_{0}^{M} c_{n} \Delta^{n} \delta(\vec{r})$ with arbitrary finite $c_{n}$

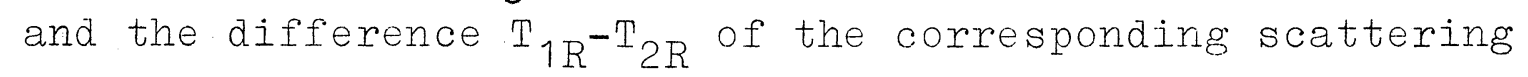
amplitudes $\mathrm{T}_{1 \mathrm{R}}$ and $\mathrm{T}_{2 \mathrm{R}}$ vanishes:

$$
T_{1 R}-T_{2 R}=\frac{1}{(2 \pi)} 3 \int_{-\infty}^{\infty} \mathrm{d} \vec{r} e^{-i \vec{p} \vec{r}} F(r) \psi(\vec{r}, k)=0
$$

in virtue of eq. $(3.4)$

- This also implies that there does not arise any arbitrariness from the scaling parameter $\Lambda$ and the length $\underset{m}{a}$ typical of potentials $V_{R}^{\lambda}{ }^{\prime}$ with $\lambda=-3-2 \mathrm{~m}$. 
In case of attractive singular potentials, $\psi(r)$ oscillates with increasing frequency as $r \rightarrow 0$ and eq. (3.4) remains no longer true. This behavior originates from the instability of the corresponding classical motion. However, we have proved that the generalized Iippmann-Sdwinger equation for $V=V^{\lambda \mu}$ also follows by continuation in $\lambda$ of the conventional LS equation (which holds for $\operatorname{Re} \lambda>-3$ ). Furthermore, we shall prove in sec.4 that the generalized IippmannSchwinger partial wave equations also follow from the conventional equations by analytic continuation in the angular momentum variable if $2 l+\lambda \neq-3-2 m$. For these continuations it is completely irrelevant whether the sign of the potential is positive or negative. Therefore, the generalized LippmannSchwinger equation (3.8) also holds for attractive singular potentials. However, the solutions will in general not be unique in that case. We see from eq.(3.7) that the schrödinger equation for attractive singular potentials is not simply given by eq.(3.1) but by the same equation with $V$ replaced by $V_{R}$. One might expect that the (repulsive) multipole core involved in the (attractive) potential $V_{R}$ renders the system stable. This, together with the fact that $\mathrm{T}_{\mathrm{R}}$ satisfies a dispersion relation ${ }^{9}$ might support the hope that a (generalized) Mandelstam representation may hold also for singular potentials notwithstanding the essential singularity of the scattering amplitude at infinite momentum.

The generalized Iippmann-Schwinger equation (3.8) is 
equivalent to a differential equation if and only if $V=V^{-\tau, 0}=g r^{-\tau}, \tau=2 n, n=1,2, \ldots$. Indeed, from eq. $(3 \cdot 9)$ we conclude that $[4] \quad\left(-\Delta_{p}\right)^{\tau / 2} V_{R}^{-\tau, \circ}(p)=g \delta(\vec{p})$ since $V_{R}{ }^{-\tau, O}(r) r^{\tau}=g$ according to eqs. $(2.7),(2.8)$. operating with $\left(-\Delta_{p}\right)^{\tau / 2}$ on eq. (3.8) the scattering amplitude $\mathrm{T}_{\mathrm{R}}$ satisfies in this case the aifferential equation

$$
\left[\left(-\Delta_{p}\right)^{\tau / 2}-\frac{g}{k^{2}-p^{2}-i \varepsilon}\right] T_{R}(\vec{p}, \vec{k})=g \delta(\vec{p}-\vec{k}) \cdot(3.10)
$$

If $g>0$, the boundary conditions are that $\mathbb{T}_{R}$ be regular at $p=0$ and vanishes sufficiently rapidly at $p=0$. If $\tau=2 n$, eq. (3.10) is of order $2 n$. If $\tau=2 n+1$, eq. (3.10) is of nonintegral order and thus is actually an integral equation. Also in the case $\mathrm{V}=\mathrm{V} \lambda \mu, \mu \neq 0, \lambda$ arbitrary, no differential equation (of finite order) can be deduced for $\mathbb{T}_{R^{\circ}}$

\section{Analytic_Continuation in_Angular Momentum}

Expanding into partial waves

$$
\begin{aligned}
& T_{R}\left(\vec{p}, \vec{p}^{\prime}\right)=\frac{1}{\sqrt{2 \pi} 2 p p^{\prime}} \sum_{\ell=0}^{\infty}(2 I+1) P_{I}\left(\hat{p} \cdot \hat{p}^{\prime}\right) f_{R I}\left(p, p^{\prime}\right) \\
& V_{R}\left(\left|\vec{p}-\vec{p}^{\prime}\right|\right)=\frac{1}{\sqrt{2 \pi} 2 p p^{\prime}} \sum_{l=0}^{\infty}(2 I+1) P_{I}\left(\hat{p} \cdot \hat{p}^{\prime}\right) F_{R I}\left(p, p^{\prime}\right)
\end{aligned}
$$

and using $(\hat{p}=\vec{p} /|\vec{p}|)$ the relation

$$
\int \alpha \omega_{q} P_{I}\left(\hat{p} \hat{q}^{\prime}\right) P_{I},\left(\hat{q} \hat{p}^{\prime}\right)=\frac{4 \pi}{2 I+1} P_{I}\left(\hat{p} \hat{p}^{\prime}\right) \delta_{I I}, \quad,
$$

we obtain from eq.(3.8) the generalized Lippmann-Schwinger 
partial wave equations

$$
f_{R I}(p, k)=F_{R I}(p, k)+\int_{0}^{\infty} \frac{F_{R I}(p, q) f_{R I}(q, k)}{k^{2}-q^{2}-i \varepsilon}(4 \cdot 3)
$$

for the partial wave amplitudes $f_{R I}$ where

$$
F_{R I}\left(p, p^{\prime}\right)=\frac{p p^{\prime}}{2 \pi} \int d \omega_{p} V_{R}\left(\left|\vec{p}-\vec{p}^{\prime}\right|\right) P_{\ell}\left(\hat{p} \hat{p}^{\prime}\right),(4 \cdot 4)
$$

Inserting the expression (2.7) into eq. (4.4) with $\varphi=(2 \pi)^{3} e^{i \vec{r}\left(\vec{p}^{\prime}-\vec{p}^{\prime}\right)}$ and using

$$
\int \alpha \omega_{p} P_{I}\left(\hat{p} \hat{p}^{\prime}\right) \int d \omega_{r} e^{i \vec{r}\left(\vec{p}-\vec{p}^{\prime}\right)}=\frac{(2 \pi)^{3}}{\sqrt{p p^{\prime}}} J_{I+1 / 2}(p r) J_{I+1 / 2}\left(p^{\prime} r\right)
$$

we obtain

$$
\begin{aligned}
F_{R I}\left(p, p^{\prime}\right)=\sqrt{\frac{p p^{\prime}}{2 r}} \int_{0}^{\infty} \operatorname{drrV}(r)\left\{J_{I+\frac{1}{2}}(p r) J_{I+1 / 2}\left(p^{\prime} r\right)\right. & (4 \cdot 5) \\
& -\sum_{n=0}^{M-1} \frac{r^{2 n+1}}{(2 n) !}\left[\frac{d^{2 n}}{d r^{2 n}}\left(\frac{J_{I+1 / 2}(p r) J_{I+1 / 2}\left(p^{\prime} r\right)}{r}\right)\right]_{r=0} \\
& -p(r) \frac{r^{2 M+1}}{(2 M) !}\left[\frac{d^{2 M}}{d r^{2 M}}\left(\frac{\left.J_{I+1 / 2}(p r) J_{I+1 / 2}\left(p^{\prime} r\right)\right)}{r}\right]_{r=0}\right\}
\end{aligned}
$$

with $\rho$ and $\mathbb{M}$ given by eqs.(2.4) and (2.10). In eq.(4.5) the subtraction terms have to be aropped if $V$ is regular, $V=V_{R}$, in which case $F_{R I}$ reduces to the familiar expression $F_{I}$,

$$
F_{I}\left(p, p^{\prime}\right)=\sqrt{\frac{p p^{\prime}}{2 \pi}} \int_{0}^{\infty} \operatorname{drrv}(r) J_{I+1 / 2}(p r) J_{I+1 / 2}\left(p^{\prime} r\right) .
$$

The subtraction terms in eq.(4.5) also vanish for $V=V \lambda \mu$ if $\operatorname{Re} I>\mathbb{M}=[-(\operatorname{Re} \lambda+3) / 2]$ : For sufficiently large angular momentum the generalized IS equation reduces to the conventional one, $F_{R I}=F_{I}, f_{R I}=f_{I}$ for $R e I>M_{\text {. Physically this is }}$ 
obvious.

If $V=V^{\lambda \mu}$, the theorem of Sec.2 tells that $F_{R I}$ is the analytic continuation in $\lambda$ of $\mathrm{F}_{1}$ from $\operatorname{Re} \lambda>-3$ to the entire $\lambda$ plane except $\lambda=-3-2 m-2 I$. If $\lambda=-3-2(I+m), F_{R I}$ is the analytic continuation of the regular part of the Laurent expansion of $\mathrm{F}_{1}$. Hence

$$
\sqrt{\frac{2 \pi}{p p^{\prime}}} F_{R I}\left(p, p^{\prime}\right)=\frac{1}{2 \pi i} \int_{C} \frac{d z}{z}\left[\int_{0}^{\infty} \operatorname{drrV}(r)\left(\frac{a_{r}}{r}\right)^{z} J_{I+\frac{1}{2}}(p r) J_{I+\frac{1}{2}}\left(p^{\prime} r\right)\right]
$$

Let us now consider $F_{1}, e q \cdot(4.6)$, as a function of the angular momentum 1. $F_{1}$ exists for $\operatorname{Rel}>-(\operatorname{Re} \lambda+3) / 2$ and can be analytically continued to the entire complex I plane except $21+\lambda=-3-2 m, m=0,1,2, \ldots$, by means of the identity

$$
\begin{aligned}
& F_{I}\left(p, p^{\prime}\right)=\sqrt{\frac{p p^{\prime}}{2 \pi^{\prime}}} \int_{0}^{\alpha} \operatorname{drrV}^{\alpha} \lambda(r)\left\{J_{I+1 / 2}(p r) J_{I+1 / 2}\left(p^{\prime} r\right)\right.
\end{aligned}
$$

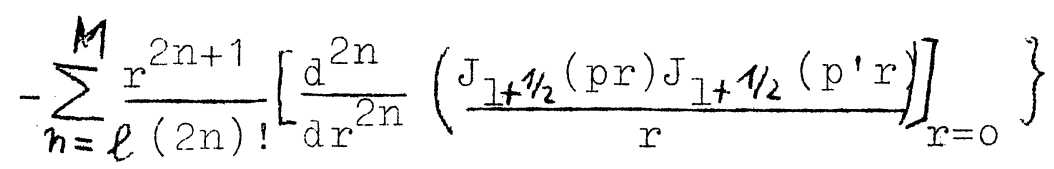

$$
\begin{aligned}
& +\sqrt{\frac{p p}{2 \pi} \int_{a}} a_{m}^{\infty} \lambda \mu(r) J_{I+1 / 2}(p r) J_{I+1 / 2}\left(p^{\prime} r\right) \\
& +\sqrt{\frac{p p}{2 \pi}} g \sum_{n=\ell}^{M} \frac{1}{(2 n) !}\left[\frac{\alpha^{2 n}}{\partial r^{2 n}}\left(\frac{J_{1+1 / 2}(p r) J_{I+1 / 2}\left(p^{\prime} r\right)}{r}\right]\right]_{r=0} \cdot B_{n \lambda \mu}
\end{aligned}
$$

valid for $\operatorname{Re}(2 I+\lambda)>-3$, where

$$
B_{n \cdot \lambda \mu}=\frac{1}{\alpha \lambda} \frac{a^{\mu}}{a \lambda^{\mu}} \frac{a^{2 n+\lambda+3}}{2 n+\lambda+3} \text {. }
$$

In virtue of eq.(2.10) the analytic continuation of the r.h.s. of eq.(4.7) coincides with the expression (4.5) if $21+\lambda \neq-3-2 \mathrm{~m}$. 
If $2 I+\lambda=-3-2 \mathrm{~m}$, eq. $(4.5)$ still is welldefined and is identical with the continuation of the regular part of the Laurent expansion of the r.h.s. of eq.(4.7). The proof goes as in Sec.2. This demonstrates that the generalized Iippmann-Schwinger partial wave equations (4.3) coincide with what one obtains by analytic continuation in the angular momentum variable of the conventional IS equations providea that $2 I+\lambda \neq-3-2 \mathrm{~m}$ and with the analytic continuation of the regular part of these equations if $2 I+\lambda=-3-2 \mathrm{~m}$. In the latter case, analytic continuation of the conventional equations themselves is not possible whereas the preceding formalism gives the correct result.

An explicit representation of $F_{R I}$ can be given for $v=v^{\lambda \mu}:$

$$
\begin{aligned}
F_{R I}\left(p, p^{\prime}\right)=g \frac{1}{\alpha^{\lambda}} \frac{a^{\mu}}{a \lambda^{\mu}}\left\{\left[H_{1, \lambda}\left(p, p^{\prime}\right)-A_{I, \lambda}\left(p, p^{\prime}\right)\right] \oplus\left(p^{\prime}-p\right)\right. \\
\left.+\left[H_{I}, \lambda\left(p^{\prime}, p\right)-A_{I, \lambda}\left(p^{\prime}, p\right)\right] \theta\left(p-p^{\prime}\right)\right\}
\end{aligned}
$$

Here, $\mathrm{H}_{1, \lambda}$ and $\mathrm{A}_{1, \lambda}$ are given by

$$
\mathrm{H}_{1, \beta}\left(\mathrm{p}, \mathrm{p}^{\prime}\right)=\frac{\mathrm{p}^{1+1 / 2} 2^{\beta+1} \Gamma\left(\frac{2 I+\beta+3}{2}\right)}{p^{1+\beta+5 / 2} \Gamma\left(-\frac{\beta}{2}\right) \Gamma(1+3 / 2)} 2^{1+\beta}\left(\frac{2 I+\beta+3}{2}, 1+\frac{\beta}{2}, 1+\frac{3}{2}: \frac{p^{2}}{p^{2}}\right)
$$

and

$$
A_{I, \lambda}\left(p, p^{\prime}\right)=\sum_{n=0}^{M} \frac{1}{(2 n) !} H_{I, \lambda+2 n+1}\left(p, p^{\prime}\right)\left[\frac{a^{2 n}}{d r^{2 n}}\left(\frac{J_{I+1 / 2}(p r) J_{I+1 / 2}\left(p^{\prime} r\right)}{r}\right)\right]_{I=0}
$$

where the term $n=\mathbb{N}$ in $A_{1} \lambda$ has to be replaced by 


$$
\frac{1}{(2 M) !}\left[\frac{d^{2 M}}{d r^{2 M}}\left(\frac{J_{I+1 / 2}(p r) i I+1 / 2\left(p^{\prime} r\right)}{r}\right)\right]_{r=0} \cdot \int_{0}^{a} d r\left(\frac{J_{I+1 / 2}(p r) J_{I+1 / 2}\left(p^{\prime} r\right)}{r}\right)
$$

if $2 I+\lambda=-3-2 \mathrm{~m}$. The last integral can be easily expressed

in terms of a series of hypergeometric functions ${ }_{2} F_{1}$ [5]

Arguments similar to those used in sec.3 show immediately that the generalized partial wave equations (4.3) hold both for repulsive and attractive singular potentials. This appears rather trivial from the point of view of analytic continuation in the angular momentum. Only in the case $V=g r^{-2 n}$ are the equations equivalent to finite order differential equations in $p$ space. The solutions are unique if the interaction is repulsive.

5. Generalized Lippmann-Schwinger Equation in Field Theory

It is an easy matter to generalize the preceding considerations to relativistic singular interactions. Examples are unrenormalizable field theories, marginally singular interactions typical of vector bosons and $\phi^{4}$ theory. In all these cases the input functions corresponding to exchanged particles are singular from the outset. In particular, in the ladder approximation to the Bethe-Salpeter equation of 
four-Fermi interactions, the interaction kernel is given by $V=\operatorname{Tr}\left(S_{F}(x) \Gamma S_{F}(x) \Gamma\right)$ and in the $\phi^{4}$ theory by $V=\left(\Delta_{F}(x)\right)^{2}$. Confining ourselves to massless exchanged particles, the input interaction kernel is of the form

$$
\mathrm{v}^{\lambda}(\mathrm{x})=g\left(\mathrm{x}_{\mathrm{r}}^{2}-\mathrm{i \varepsilon}\right)^{\lambda}
$$

with $\lambda \leqslant-1$, the case $\lambda=-3$ being characteristic for leptonlepton scattering due to the exchange of massless lepton pairs.

Singular interaction kernels such as $V^{\lambda}$ are purely formal quantities arising from an evaluation of Feynman diagrams. They suffer from a similar lack of definition on the light cone as do singular potentials at the origin. In analogy to the nonrelativistic case we can give a meaning to these kernels by generalizing Feynman's prescription to singular input functions. There are various equivalent ways to do this 7 , 6], the simplest one being analytic continuation. Let us consider the formal interaction kernel $\mathrm{V}^{\lambda}$, eq. (5.1), and let $\varphi$ be a test function. Then we may associate with $\mathrm{V}^{\lambda}(\mathrm{x})$ the generalized Feynman kernel $V_{\mathrm{R}}^{\lambda}(\mathrm{x})$ by requiring that the relation

$$
\int_{-\infty}^{\infty} d^{4} x V_{R}^{\lambda}(x) \varphi(x)=\int_{-\infty}^{\infty} a^{4} x v^{\lambda}(x) \varphi(x)
$$

holds for all those $\varphi(x)$ which vanish sufficiently strongly on the light cone. If $\varphi$ is any test function, not necessarily vanishing on the light cone, then the integral on the roh.s. of eq.(5.2) exists for $\operatorname{Re} \lambda>-1$ and can be continued analytically to the entire complex $\lambda$ plane except $\lambda=-n, n=2,3, \ldots$, where it has simple poles. In complete analogy to the nonrelativistic 
case (cf., Sec.2) it follows that the generalized Feynman kernel $V_{R}^{\lambda}(x)$ associated with the formal kernel $V^{\lambda}(x)$ is given by the relation

$$
\int_{-\infty}^{\infty} d^{4} x V_{R}^{\lambda}(x) \varphi(x)=a \cdot c \cdot \int_{-\infty}^{\infty} a^{4} x v^{\lambda}(x) \varphi(x)
$$

where the symbol a.comeans analytic continuation of the integral on the r.h.s. of eq. (5.3) from $\operatorname{Re} \lambda>-1$ to the entire complex $\lambda$ plane except $\lambda=-n(n=2,3, \ldots)$, and analytic continuation of the regular part of the Laurent expansion around $\lambda=-n$ if $\lambda=-n(n=2,3, \ldots)$. This prescription can be summarized into the formula (cf., also sec.2)

$$
\int_{-\infty}^{\infty} d^{4} x V_{R}(x) \varphi(x)=\frac{1}{2 \pi i} \int_{c} \frac{d z}{z}\left[\int_{-\infty}^{\infty} d^{4} x V(x)\left(\frac{a^{2}}{x_{r}^{2}-i \varepsilon}\right)^{z} \varphi(x)\right]
$$

This formula holds for $V=V^{\lambda}, V_{R}=V_{R}^{\lambda}$ and should be taken as a definition of the generalized Feymman kernel $V_{R}$ associated with any formal singular interaction kernel $V$. The constant a is an arbitrary parameter with dimension of length. In eq. (5.4) one first has to perform the $\mathrm{x}$ integration on the r.h.s. for Re z sufficiently large negative where the integral converges, then continue the resulting function of $\mathrm{z}$ analytically to a region containing the origin $z=0$ and finally perform the $z$ integration alorg a small circle $C$ around $z=0$ 。 The resulting Feynman kernel $V_{R}$ is independent of the arbitrary length $\underset{m}{a}$ if $V$ does not behave as $\left(x_{y}^{2}-i \varepsilon\right)^{-n}, n=2,3, \ldots$ on the light cone $x_{r}^{2}=0$. Physically speaking, the length $\underset{m}{a}$ enters if and only if the integral $\int \mathrm{d}^{4} \mathrm{x} V(\mathrm{x}) \varphi(\mathrm{x})$ diverges logarith- 
mically near the light cone. The above definition of generalized Feynman kernels obviously can be extended to pseudoeuclidean spaces of arbitrary dimension. By the method of complex extension ${ }^{7}$-- which in some cases is equivalent to Euclidean rotation -- it follows, then, that the potential $\mathrm{V}_{\mathrm{R}}(r)$ equivalent to $\mathrm{V}(r)$ discussed in Sec.2 is just a particular case of the generalized Feynman prescription introduced above. In a forthcoming paper we shall deduce the generalized Feynman prescription from general principles of field theory ${ }^{[6]}$ It should be pointed out that, in general, the formal interaction kernel $V$ involves products of propagators of equal arguments, $\mathrm{V}(\mathrm{x})=\mathrm{S}_{\mathrm{F}}(\mathrm{x}) \mathrm{S}_{\mathrm{F}}(\mathrm{x})$ or $\mathrm{V}(\mathrm{x})=\left[\triangle_{\mathrm{F}}(\mathrm{x})\right]^{2}$ etc。. The right hand side of eq.(5.4) automatically eliminates all difficulties associated with such products in virtue of the term $\left(a^{2} /\left(x_{v}^{2}-i \varepsilon\right)\right)^{z}$, Re $z$ sufficiently large negative。

From eq. (5.3) or (5.4) we obtain immediately the Fourier transform $V_{R}(p)$ of $V_{R}(x)$ by substituting exp(ipx) for $\varphi(x)$. Using Bochner's formula, nowadays known as the reduction formula of Feinberg and Pais 3, 7,10,

$$
\int_{-\infty}^{\infty} d^{4} x f\left(x_{r}^{2} \pm i \varepsilon\right) e^{i p_{v} x_{V}}=\mp \frac{i(2 \pi)^{2}}{\left(p^{2} \mp i \varepsilon\right)^{1 / 2}} \int_{0}^{\infty} d r r^{2} J_{1}\left(r\left(p_{V}^{2} \mp i \varepsilon\right)^{1 / 2}\right) f\left(r^{2}\right)
$$

( $J_{1}$ being a Bessel function) we find

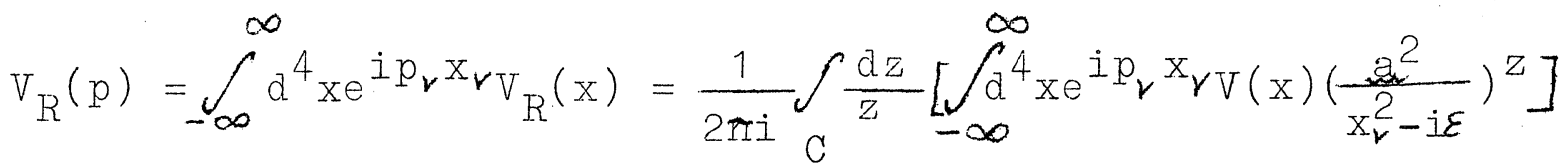

$$
\begin{aligned}
& =2 \pi \int_{C} \frac{d z}{z} \frac{1}{\left(p_{r}^{2}+i \varepsilon\right)^{1 / 2}}\left[\int_{0}^{\infty} d r r^{2} J_{1}\left(r\left(p^{2}+i \varepsilon\right)^{1 / 2}\right) V\left(r^{2}\right)\left(\frac{a^{2}}{r^{2}}\right)^{z}\right]
\end{aligned}
$$


where $r$ is a one-dimensional, Euclidean variable. In particular, for $\mathrm{V}=\mathrm{V}^{\lambda}$ we obtain

$$
V_{R}^{\lambda}(p)=g i(4 \pi)^{2}{ }_{4}^{\lambda} \Gamma(\lambda+2)\left(p_{r}^{2}+i \varepsilon\right)^{-\lambda-2} / \Gamma(-\lambda)
$$

if $\lambda \neq-n, n=2,3, \ldots$ and

$$
V_{R}^{\lambda}(p)=\frac{g(-1)^{n-1}(4 r)^{2} i\left(p_{v}^{2}+i \varepsilon\right)^{n-2}}{(n-1) !(n-2) ! 4^{n}}\left[\log \left(\frac{a_{m}^{2}\left(p_{v}^{2}+i \varepsilon\right)}{4}\right)-\psi(n)-\psi(n-1)\right.
$$

if $\lambda=-n, n=2,3, \ldots$, where $\psi(z)=\Gamma^{\prime}(z) / \Gamma(z) \cdot \mathrm{Eq} \cdot(5.7)$ also follows from eq.(5.3) with $\varphi=\exp \left(i_{V Y}^{p_{V}}\right)$ by analytic continuation. If $\lambda=-n, V_{R}^{\lambda}(p)$ depends on the arbitrary length $\underset{m}{a}$ which may be chosen as $\underset{m}{a}=\Lambda \mathrm{g}^{1 / \eta}$, $\Lambda$ being an arbitrary scaling parameter. Again we observe that $V_{R}^{-n}(x)$ is not scaling invariant if $\underset{m}{a}$ is kept fixed, scalirg invariance being reestablished if $\underset{\mathrm{m}}{\mathrm{m}}$ is left aroitrary.

Let us now consider tre Bethe-Salpeter scattering wave function $\psi(x)=\psi(x, p, E)$ for the relative motion $\left(x=x_{1}-x_{2}\right)$ of two fermions either of zero nass $(m=0)$ or with vanishing com, energy E。 More general interaction processes can be dealt with in the same manner. The free two-partiole Green's function $G$ is then given by

$$
G\left(x-x^{\prime}\right)=\int_{-\infty}^{\infty} a^{4} x^{\prime \prime} S_{F}\left(x-x^{\prime \prime}\right) S_{F}\left(x^{\prime \prime}-x^{\prime}\right)=\Delta_{F}\left(x-x^{\prime} ; x\right)
$$

suppressing spin indices and $\gamma$ factors. Here, $K=E / 2$ in case of zero mass particles and com。energy $\mathrm{E} \neq \mathrm{O}$, and $\boldsymbol{x}=\mathrm{m}$ in case of particles of mass $m$ and vanishing com. energy. Then the 
Bethe-Salpeter equation in the ladder approximation becomes 3,5

$$
\psi(x)=e^{i p_{v} x_{v}+2 i} \int_{-\infty}^{\infty} d^{4} x^{\prime} G\left(x-x^{\prime}\right) V_{R}\left(x^{\prime}\right) \psi\left(x^{\prime}\right)
$$

where the generalized Feynman kernel $V_{R}$ has been used as input rather than the formal kernel $V$. Contrary to the case of repulsive singular potentials this replacement cannot be justified by appealing to eq.(5.2) with $\varphi=G \psi$ since $\psi(x)$ in general does not vanish on both sides of the light cone. Instead, the replacement of $V$ by $V_{R}$ in the Bethe-Salpeter equation is made possible by the causal factor is in the kernels. This factor makes the equations well-defined both in the repulsive and attractive case. Moreover, the formal solution of eq.(5.9) is identical with the solution of the same equation with $V_{R}$ replaced by $V$ in virtue of the is in the kernels. It is (within the present context) only the need for a Fourier transform which requires the introduction of $V_{R}$ in place of $V$-- and, of course, the question for making things existent in the rigorous sense.

Defining the off shell amplitude $\mathrm{T}_{\mathrm{R}}$ by

$$
T_{R}\left(p, p^{\prime}, E\right)=\int_{-\infty}^{\infty} d^{4} x e^{-i p p^{\prime} x_{v V_{R}}(x) \psi(x, p, E)}
$$

and operating on both sides of eq.(5.9) with $V_{R} \exp \left(-i p_{v}^{\prime} x_{v}\right)$ we obtain the generalized Lippmann-Schwinger equation for the $T$ matrix of fermion-fermion scattering (within the approximation considerea)

$$
T_{R}\left(p, p^{\prime}, E\right)=V_{R}\left(p-p^{\prime}\right)+\frac{1}{(2 \pi)^{8}} \int_{-\infty}^{\infty} d^{4} q \frac{V_{R}\left(q-p^{\prime}\right) T_{R}(p, q, E)}{q^{2}-x^{2}+i \varepsilon}
$$


where $V_{R}(p)$ exists (contrary to $V(p)$ ) according to eq. $(5.6)$. $T_{R}$ becomes the physical scattering arnplitude if $p$ and $p^{\prime}$ are taken to have their physical values for elastic scattering, $p^{2}=p^{2}, p^{2}+m^{2}=E^{2} / 4$.

The Bethe-Salpeter equation (5.9) is equivalent to the differential equation

$$
\left(\square_{\mathrm{x}}+x^{2}-4 \mathrm{~V}_{\mathrm{R}}(\mathrm{x})\right) \psi(\mathrm{x})=0 .
$$

Singular equations of this type 11 play a role also in connnection with Goldstone particles 12 . On the other hand, the generalized Iippmann-Schwinger equation (5.11) reduces to a finite order differential equation only if

$$
\begin{aligned}
V=V^{-n}= & g\left(x_{v}^{2}-i \varepsilon\right)^{-n}, n=1,2,3, \ldots: \\
& {\left[\left(-\square p^{\prime}\right)^{n}+\frac{g /(2 \pi)^{4}}{p^{\prime 2}-x^{2}+i \varepsilon}\right] T_{R}\left(p, p^{\prime}, E\right)=(2 \pi)^{4} g \delta^{4}\left(p-p^{\prime}\right) }
\end{aligned}
$$

in virtue of $\left(\square_{p}\right)^{n} V_{R}^{-n}(p)=g(-1)^{n}(2 \pi)^{4} \delta^{4}(p)$. [4]

Suppose now that a rotation to Euclidean metric can be done in $e q .(5.9)$ and that the resulting Euclidean interaction kernel is purely repulsive. In that case, the Euclidean rotation just projects out either space-like or time-like domains. Then eq. (5.9) passes into an equation identical with eq.(3.1) except for a modified Green's function $G, \vec{r}$ being now a four-dimensional Euclidean variable. Since the interaction is repulsive, the relation (3.4) holds true and permits one to deduce at once the relativistic generalized Iippmann-Schwinger equation ( 5 o1) (in Euclidean space) from first principles. Actualiy, eq.(5.11) operates in Minkowski $65 / 1211 / 5$ 
space, and continuation in $\lambda$ or in the angular momentum variable may again be applied to show that the equation holds also for attractive forces. Whether the equation has a solution in that case is of course an open question. Our previous arguments as to scaling properties and the intervention of an arbitrary phase in the attractive case remain unaltered.

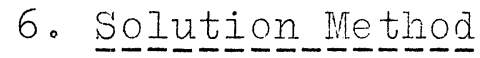

From the behavior of the kernels $V_{R}(k)$, eqs. $(2.14,15)$, and $V_{R}(p)$, eqs. $(5.7,8)$, we see that the generalized IippmannSchwinger equations $(3.8)$ and $(5.11)$ and the partial wave equations (4.3) are not of Freaholm type if the interaction is singular. Various methods for solving such equations in coordinate space have recently been discussed $4,5,6$

In terms of the integral representatior of $V_{R}(p)$,

$$
V_{R}(p)=\frac{1}{2 \pi i} \int_{C} \frac{d z}{z}\left[\int_{-\infty}^{\infty} a^{4} x e^{i p_{r} x_{V}}(x)\left(\frac{a^{2}}{x_{r}^{2}-i \varepsilon}\right)^{z}\right]
$$

a simple iteration scheme may be set up in analogy to a method devised in Ref. 5: Let us insert the representations $(2.17)$ and $(5.6)$ into the Iippmann-Schwinger equations (3.8) and (5.11), respectively. Assuming Re $z$ sufficiently large negative at each step, we obviously can solve the resulting 
equations by simple iteration. This iterative solution is given by

$$
T_{1}=\sum_{n=0}^{\infty} g^{n} T_{1 n}\left(p, p^{\prime}, T^{n}\right)
$$

where

$$
T_{1 n}=\frac{1}{2 \pi i} \int_{C} \frac{d z}{z} f_{n}\left(z ; p, p^{\prime}, E\right)
$$

is the analytic continuation to $z=0$ of a well-defined function $f_{n}$ or the continuation of its regular part. However, this iterative solution certainly is not the exact solution to the Iippmann-Schwinger equation, for the series $(6.2)$ of analytic continuations is (in general) different from the analytic continuation of the series, $(2 \pi i)^{-1} \sqrt{\frac{a}{z}} \frac{\sum_{0}^{\infty} g^{n} f_{n}}{}$. Looking at the difference $\mathrm{T}_{\mathrm{R}}-\mathrm{T}_{1}=\mathrm{AT}_{2}$ between the exact solution $T_{R}$ and the iterative solution $T_{1}$ one finds that $\mathrm{T}_{2}$ satisfies an iterable integral equation too and the exact solution is given by

$$
\mathrm{T}_{\mathrm{R}}=\mathrm{T}_{1}+A \mathrm{~T}_{2}
$$

where $A=-\lim _{p^{\prime \rightarrow \infty}}\left(T_{1} / T_{2}\right)$ can be expressed in terms of $T_{1}$ alone and in general is nonanalytic at zero coupling. In case of potential scattering one finds for $V=V^{\lambda}$ the partial wave amplitudes $f_{R I} \simeq \exp \left(-c(g) p^{1+2 / \lambda}\right)$ as $p \rightarrow \infty$ with $\operatorname{Re} c(g)>0$ for $g>0$. For relativistic fermion-fermion scattering via massless fermion pairs, eq. (5.9) with $V=g\left(x_{v}^{2}-i \varepsilon\right)^{-3}$ is solved in the case $p_{v}=0$ by $\psi(x)=\exp \left(-\sqrt{g} /\left(x_{v}^{2}-i \varepsilon\right)\right)$ and $\mathrm{T}_{\mathrm{R}}$ can be calculated by means of eq.(6.5). We shall discuss this method elsewhere in connection with singular $\mathrm{N} / \mathrm{D}$ equations [6] 


\section{Acknowledgments}

One of us (E.P.) wishes to thank the Deutsche Forschungsgemeinschaft for a research grant。 The other (W.G.) is grateful to Drs. H. Cornille, A.P. Contogouris and M.B. Halpern for stimulating discussions. He wishes to thank Profs.V. Weisskopf and $I$. Van Hove for the warm hospitality of the Theoretical Division at CERN. 


\section{Reference-}

1 B.A. Iippmann and J. Schwinger, Phys.Rev。 79,469(1950)

2 IN.IN。Khuri and A. Pais, RevoMod.Phys.36,590(1965)

3 R.Sawyer, Phys.Rev。134, B448(1964)

G.Feinberg and A.Pais, Phys.Rev.131, B 2724 (1963); Phys.Rev。133, B 474 (1964)

G.Domokos, Acta Phys.Austriaca Suppl.I, 1965

4 HoCornille, Nuovo Cimento in press and CERN preprints $1964 / 65$

F. Calogero, Nuovo Cimento 37, 756,76o (1965) and University of Roma preprints。 W.Guttinger, R.Penzl and E.Pfaffelhuber, Nuovo Cimento 35, 1247 (1965)。

A complete list of references may be found in these papers.

5 W.Guttinger,R.Penzl and E.Pfaffelhuber, Annals of Physics (NoI。) in presso

6 B.A.Arbuzov and A.T.Pilippov, Phys.Letters 13,95 (1965) and Dubna preprints

7 W.Guttinger, Fortschr.d.Physik, in press; Nuovo Cimento 10,1 (1958), Nucl.Phys。 9, 429 (1959)。 B. Schroer and K. Bardakci, Univ.of Pittsburgh preprint 1965.C.f.also I.M.Gel'fand and G.E.Shilov, Generalized Functions (New York 1964); I.Schwartz, Théorie des 
distributions (Paris 1951)。

8 0. Brander, Phys.Letters 4,218 (1963)

9 A. Martin, to be published

10 C.Bollini., J。Giambiagi and A.Gonzalez Dominguez,

J.Math。Phys。ㅁ, 165 (1965)

11 J.Goldstein, Phys.Rev.91, 1516 (1953),

N.N. Nakanishi, Phys.Rev。, in press

12 M. Baker, K. Johnson and B.W. Lee, Phys.Rev.133, B 209(1964)

\section{토으으므으트모}

[1] A test function is an infinitely differentiable function having compact support or behaving at infinity such that $V \varphi$. is integrable there.

[2] The need for the factor $p$ is easily seen from eq.(2.7).

[3] In potential scattering theory, $V$ is called singular if $\operatorname{Re} \lambda<-2$ because then the schrödinger equation cannot be solved by iteration. The difference in the definition "singular" ( $\operatorname{Re} \lambda<-2$ or $\operatorname{Re} \lambda \leqslant-3$ ) originates from a factor $1 / \mathrm{r}$ produced by $G$ in eq.(3.1). In the present context we call a potential singular if it does not have a Fourier transform.

[4] Note that this relation does not hold for $V$.

[5] It is easily seen from these representations how the pole terms, which would arise in $\mathrm{F}_{1}$ for $21+\lambda=-3-2 \mathrm{~m}$, are cancelled in eq. $(4.5)$. For example, if $\mu=0,1=0$, 
the term in $\mathrm{F}_{1}$ which would diverge for $\lambda=-3$

is replaced in $\mathrm{F}_{\mathrm{Rl}}$ by $\sqrt{\mathrm{pp}} \log \left(\mathrm{p}^{2} / \mathrm{p}^{\prime 2}\right)$ 。

[6] W.Gittinger and E.Pfaffelhuber, to be published. 